\title{
MAPPING SETS AND HYPERSETS INTO NUMBERS
}

\author{
GIOVANNA D'AGOSTINO, EUGENIO G. OMODEO, ALBERTO POLICRITI, \\ AND ALEXANDRU I. TOMESCU
}

\begin{abstract}
We introduce and prove the basic properties of encodings that generalize to non-well-founded hereditarily finite sets the bijection defined by Ackermann in 1937 between hereditarily finite sets and natural numbers.
\end{abstract}

Key words: Computable set theory, non-well-founded sets, bisimulation, Ackermann bijection.

\section{INTRODUCTION}

"Consider $\cdots$ all the sets that can be obtained from $\emptyset$ by applying a finite number of times the operations of forming singletons and of forming binary unions; ... the class of all sets thus obtained $\cdots$ coincides with the class of what are called in set theory the hereditarily finite sets, or sets of finite rank. ... We readily see that $\cdots$ we can establish a one-one correspondence between natural numbers and hereditarily finite sets". This passage of [TG87, p. 217] refers to a specific correspondence, whose remarkable properties were first established in Ackermann's article [Ack37]. Among other virtues, Ackermann's bijection enables one to retrieve the full structure of a hereditarily finite set from its numeric encoding by means of a most simple recursive routine deep-rooted in the binary representation of numbers. To be more specific about this, let us call $i$-th set the hereditarily finite set whose encoding is $i$; then it holds that the $j$-th set belongs to the $i$-th set if and only if the $j^{\text {th }}$ bit in the base- 2 representation of $i$ is 1 . At a deeper level, Ackermann's bijection gives an insight on why "the Peano arithmetic and the extended Zermelolike theory of finite sets are definitionally equivalent, and therefore equipollent in means of expression and proof" [TG87, p. 225].

What happens when one considers a richer collection of hereditarily finite sets, over which membership forms cycles? This paper adjusts Ackermann's bijection to a specific collection $\mathrm{HF}^{1 / 2}$ of sets of this nature, drawn from Aczel's universe of hypersets [Acz88, BM91]. Traditional sets, HF, will retain the same images as before in a first bijection we will propose; together with those images, which span all natural numbers, the images of the new, 'ill-founded' hereditarily finite sets will span the set of all dyadic rationals. The choice of this numeric domain stems from the rationale that we want the membership relation to be readable, as before, from the binary representation of numbers.

Our proposed extension will result from a natural move: we will construct both correspondences, Ackermann's and our own, via a splitting technique borrowed from algorithmics [PT87, DPP04].

Much like Ackermann's bijection which it generalizes, the bijection to be constructed in this paper encodes a most basic data type by numbers. This embedding

Date: November 16, 2015. 
acts as an Occam's razor, by reducing multiplicity to simplicity: in the case of HF-sets, one can implement a full battery of set-handling methods by resorting to natural numbers as their internal representation [CNACO08]; likewise, one can implement $\mathrm{HF}^{1 / 2}$-sets on top of rational numbers via the encoding technique to be proposed below. This paper does not directly address the issue of manipulating set/hypserset constraints in which variables can occur, but we see investigations like the one presented here as unavoidable preparatory steps in sight of improved methods of computational logic that embody some set-theoretic ability. ${ }^{1}$

We will conclude our paper by proposing another mapping of hypersets into, this time, real numbers. This further encoding bears a different-more formalsimilarity with Ackermann bijection, as it is obtained from it by a change of sign in Ackermann's original formula. In spite of its simple definition, the basic properties of our second encoding become rather delicate to prove and are only initially tackled here.

Organization of the paper. After revisiting, in Sec. 1, the Ackermann order $\prec$ of $\mathrm{HF}$ and discussing the possibility of extending it to $\mathrm{HF}^{1 / 2}$, in Sec. 2 we give a closer look at $\prec$, so as to get an alternative characterization of it through an algorithmic splitting technique. In Sec. 3, the very same splitting technique enables us to extend Ackermann's ordering to $\mathrm{HF}^{1 / 2}$ naturally. In Sec. 4 we use the extended ordering to define the function $\mathbb{Z}_{A}$ giving integer positions to the hereditarily finite hypersets, and then present the encoding $\mathbb{Q}_{A}$ of $\mathrm{HF}^{1 / 2}$ by means of dyadic rationals. Sec. 5 is devoted to the study of the simple variant obtained from Ackermann's mapping by changing sign in the exponent of the original Ackermann's formula.

Related Work. The splitting technique was already used in [LS99] to get a linear ordering of $\mathrm{HF}^{1 / 2}$, a main requirement being, there, that the resulting order should be definable by means of a formula of First-Order Logic with Fixed Points. Our goal here is different, as we seek a natural correspondence between hypersets and numbers which induces, by a restriction, Ackermann's ordering of HF. As remarked by the authors of [LS99], it turns out that the linear order proposed in that paper is not such an extension.

\section{Basics, Preliminary Observations, and Outline}

1.1. General outlook. When, as it seldom happens, a novel notion acquires significance in various branches of mathematics at the same time, that pervasive notion gradually slides down towards the first principles and it candidates for a preeminent role in the foundations of mathematics. This happened when, in the 1920s, RECURSION gained ground as a convenient way of hooking the specifications of functions and relations of domain $V$ to a dyadic relation $E$ that meets, on $V$, suitable conditions. This happened again in the 1980s, when BISIMILARITY imposed itself as a ubiquitous equivalence criterion for partitioning a class $V$ in a way that again relies on a dyadic relation $E$ on $V$. In 1925 von Neumann managed to tie recursion directly to set membership by introducing a new axiom, regularity [vN25], among

\footnotetext{
${ }^{1}$ Methods of this more advanced kind can be found, e.g., in [OP95, DOP99, ADR99, DPR06, DPR08, Dov14]. The algorithmic challenges they pose generally stem from various complexity sources, a few of which are best isolated in the variable-free context addressed in this paper.
} 
the postulates of the Zermelo-Fraenkel set theory ZF. Likewise, around 1985, Aczel investigated the consequences of superseding regularity by an anti-foundation axiom: $A F A\left[\right.$ Acz88]. ${ }^{2}$ While disrupting the hierarchical structure of von Neumann's universe of sets by enriching it with a host of new entities (at times called 'hypersets' [BM91]), AFA also avoids overcrowding the universe, by enforcing bisimilarity as a criterion for equality between sets.

Concerning the former of these issues, define a system $\mathcal{M}=(V, E)$ to consist of a class $V$ of NODES paired with a class $E \subseteq V \times V$ of ARCS ${ }^{3}$ such that

the 'children' $u 户=_{\text {Def }}\{v: v \in V \wedge u E v\}$ form a set, for each node $u$.

Then, if the WELL-FOUNDEDNESS condition

$$
\forall w(w \subseteq V \wedge w \neq \emptyset \Longrightarrow \exists m \in w(m \text { } \cap w=\emptyset))
$$

is met, namely if every nonnull set $w$ of nodes has a 'minimal' element $m$ relative to the converse ${ }^{4} E^{-1}$ of $E$, we can plainly specify recursive functions over $V$. This is why we can do recursion over the class of all finite sets (or over any subclass of it, e.g. over the set of natural numbers): finite sets form, in fact, the largest subclass $V$ of the universe of all sets that is closed with respect to the strict inclusion relation $\supsetneq$ and that meets, relative to this $E$, the well-foundedness condition. If we assent to von Neumann's regularity axiom, by which $\in$ is well-founded, then we can exploit recursion even more globally, by taking as $V$ the class of all sets and, as $E$, the converse $\ni$ of membership. To see the ensuing powerful mechanism at work, suffice it to consider the following $\in$-recursive definitions of a HEREDITARILY FINITE set (based on any reasonable predicate ${ }^{5}$ Is_finite), as well as the definitions of RANK and TRANSITIVE CLOSURE (the set of ultimate members) of any set $X$ :

$$
\begin{gathered}
\operatorname{HF}(F) \Leftrightarrow \Leftrightarrow_{\text {Def }} \text { Is_finite }(F) \wedge \forall y \in F \mathrm{HF}(y) ; \\
\operatorname{rk}(X)={ }_{\text {Def }} \sup \{\operatorname{rk}(y)+1: y \in X\} ; \\
\operatorname{TrCl}(X)={ }_{\text {Def }} X \cup \bigcup\{\operatorname{TrCl}(y): y \in X\},
\end{gathered}
$$

where the usual definition $\bigcup W={ }_{\text {Def }}\{z: \exists y(y \in W \wedge z \in y)\}$ applies.

Other useful notions definable recursively (their rationale being regularity again) are the following, where $F$ and $F^{\prime}$ are restrained to be hereditarily finite sets:

$$
\begin{aligned}
\mathbb{N}_{A}(F) & =\sum_{\text {Def }} \sum_{h \in F} 2^{\mathbb{N}_{A}(h)}, \\
F \prec F^{\prime} & \Leftrightarrow \Leftrightarrow_{\text {Def }} \max _{\prec}\left(F \backslash F^{\prime}\right) \prec \max _{\prec}\left(F^{\prime} \backslash F\right) .
\end{aligned}
$$

This $\mathbb{N}_{A}$ is a noticeable bijection, discovered by Wilhelm Ackermann around 1937 (see [Ack37, Lev79]), between the hereditarily finite sets and $\mathbb{N}$; its very existence proves that the property HF delimits a set. As for $\prec$, this is a strict ('anti-lexicographic') ordering of all hereditarily finite sets, ${ }^{6}$ which extends, and is

\footnotetext{
${ }^{2}$ [BM96, p. 5] indicates [FH83] as a precursor of Aczel's set theory.

${ }^{3} \mathrm{~A}$ system is also named a GRAPH when its nodes form a set, as opposed to a proper class.

${ }^{4}$ The CONVERse $E^{-1}$ of a dyadic relation $E$ is, by definition, the class $\{\langle v, u\rangle: u E v\}$; the COMPOSITION of $E$ with another dyadic relation $E^{\prime}$ is $E \circ E^{\prime}{ }_{{ }_{\text {Def }}}\left\{\langle x, z\rangle: \exists y\left(x E y \wedge y E^{\prime} z\right)\right\}$.

${ }^{5}$ E.g. Is_finite $(F) \Leftrightarrow \Leftrightarrow_{\text {Def }} \forall x((x \neq \emptyset \wedge \forall y \in x(y \subseteq F)) \Longrightarrow \exists m \in x \forall z \in x(z \subseteq m \Longrightarrow z=m))$ after Tarski, see [Tar24].

${ }^{6}$ Let, by convention, $\max \prec \emptyset \prec d$ hold for any set $d \neq \emptyset$; thus $F \prec F^{\prime}$ will hold when $F \subsetneq F^{\prime}$.
} 

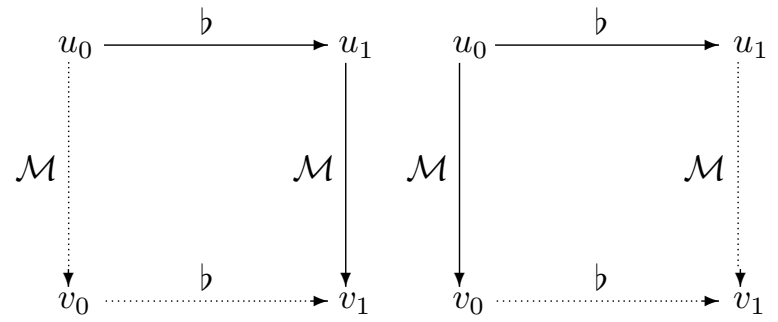

Figure 1. Properties of a bisimulation $b$ on $\mathcal{M}$

isomorphic to, the standard ordering of $\mathbb{N}$. Actually, the biimplication $F \prec F^{\prime} \Leftrightarrow$ $\mathbb{N}_{A}(F)<\mathbb{N}_{A}\left(F^{\prime}\right)$ holds whenever both $\operatorname{HF}(F)$ and $\operatorname{HF}\left(F^{\prime}\right)$ hold.

To now report about Aczel's proposal cited at the beginning, we must again consider a system $\mathcal{M}=(V, E)$. Define a Bisimulation on $\mathcal{M}$ to be a relation $b \subseteq V \times V$ that satisfies the inclusions

$$
b \circ E \subseteq E \circ b, \quad E^{-1} \circ b \subseteq b \circ E^{-1} ;
$$

i.e., $b$ is a bisimulation if and only if $u_{0} b u_{1}$ implies, for $j=0,1$, that to every child $v_{j}$ of $u_{j}$ there corresponds at least one child $v_{1-j}$ of $u_{1-j}$ such that $v_{0} b v_{1}$ holds (cf. Fig. 1).

Then let BISIMILARITY to be the relation $\equiv_{\mathcal{M}}$ defined between nodes $u, v$ as follows:

$$
u \equiv \mathcal{M} v \Leftrightarrow_{\text {Def }} u b v \text { holds for some small bisimulation } b
$$

('small' meaning that b must be a set, not a proper class, of pairs of nodes). It plainly turns out that $\equiv_{\mathcal{M}}$ is a bisimulation (actually, the largest of all bisimulations) on $\mathcal{M}$; moreover, it is an equivalence relation over $V$.

Let $\mathcal{U}$ be the system whose nodes are all the sets and whose arcs are the pairs $\langle u, v\rangle$ of sets such that $v \in u$. Aczel's and von Neumann's view of sets agree on a crucial 'parsimony' criterion: $u \equiv \mathcal{U} v$ implies that $u$ and $v$ are the same set; consequently, if we consider the graph $\mathcal{U}_{x}$ issuing from any set $x$ (so that every node of $\mathcal{U}_{x}$ is reachable from $x$ through a chain of $\ni$-arcs), we can be sure that $u \equiv \mathcal{U}_{x} v$ never holds between distinct nodes $u, v$ of $\mathcal{U}_{x}$-in particular, no two nodes have the same children. The two views differ radically, though, on the decision about what shapes a graph $\mathcal{U}_{x}$ can take. Aczel imposes no restrictions on $\mathcal{U}_{x}$ but the ones just recalled (each node must be reachable from the root $x$, no two nodes are bisimilar); after von Neumann, $\mathcal{U}_{x}$ must be devoid of infinite paths.

Aczel's universe of sets - to which most of our subsequent discussion will referhence encompasses von Neumann's celebrated cumulative hierarchy. ${ }^{7}$ Its greater richness eases the modeling of circular phenomena, with special success when bisimilarity is at work, as it happens with automata, Kripke structures, communicating systems (cf. [DPP04]). But many familiar definitions need to be reformulated for Aczel's sets.

\footnotetext{
${ }^{7}$ In its turn, as explained in [Acz88, Chapter 3], the construction of Aczel's universe of sets can be performed inside von Neumann's universe. For an adaptation of that 'inner' construction to the narrower universe of the hereditarily finite sets, cf. [OP95].
} 
In particular, $\mathrm{HF}$, a.k.a. $\mathrm{HF}^{0}$, has two ill-founded variants under AFA (cf. [BM96, p. 268]):

$$
\mathrm{HF}^{0} \subsetneq \mathrm{HF}^{1 / 2} \subsetneq \mathrm{HF}^{1}
$$

where

$$
\begin{aligned}
\mathrm{HF}^{0}(F) \Leftrightarrow & \Leftrightarrow \\
& \forall w \subseteq \operatorname{TrCl} \text { Is_finite }(\operatorname{TrCl}(F)) \wedge \\
\operatorname{HF}^{1 / 2}(F) & \Leftrightarrow \Leftrightarrow_{\text {Def }} \text { Is_finite }(\operatorname{TrCl}(F)), \\
\operatorname{HF}^{1}(F) & \Leftrightarrow \Leftrightarrow_{\text {Def }} \forall y \in \operatorname{TrCl}(\{F\}) \text { Is_finite }(y),
\end{aligned}
$$

and the following - suitably recast - notion of $\mathrm{TrCl}$ applies:

$$
\operatorname{TrCl}(X)={ }_{\text {Def }} \bigcup_{n \in \mathbb{N}} X \gg_{n},
$$

where $X 户_{0}={ }_{\text {Def }} X$ and $X 户_{i+1}={ }_{\text {Def }} \bigcup\left(X \longmapsto_{i}\right)$.

Either one of $\mathrm{HF}^{1 / 2}$ and $\mathrm{HF}^{1}$ comprises, in addition to what belongs to $\mathrm{HF}$, an infinitude of hypersets; but the former is still a countable set, whereas $\mathrm{HF}^{1}$ is uncountable. This partly explains why the sets in $\mathrm{HF}^{1 / 2}$ are said to be rational while the ones in $\mathrm{HF}^{1}$ are called irrational hereditarily finite sets. Limiting our study to the former in this paper, we will see that $\mathrm{HF}^{1 / 2}$ has a natural counterpart in the set $\mathbb{Q}_{2}^{+}=\left\{\frac{n}{2^{m}}: n, m \in \mathbb{N}\right\}$ of the so-called (non negative) dyadic rational numbers, very much like HF has its natural counterpart in $\mathbb{N}$. Specifically, we will extend Ackermann's bijection $\mathbb{N}_{A}: \mathrm{HF} \longrightarrow \mathbb{N}$ into a bijection $\mathbb{Q}_{A}: \mathrm{HF}^{1 / 2} \longrightarrow \mathbb{Q}_{2}^{+}$.

1.2. Hypersets as graphs. It will be convenient to look at hereditarily finite hypersets also from a different angle, by focusing on the properties of the membership graph underlying the transitive closure of each of them.

Definition 1. A pointed graph $(G, v)$ is a graph $G=(V, E)$ with a distinguished node $v \in V$ (its point) from which every node in $V$ is $E$-reachable.

Definition 2. Given a set $S$, we denote by $G_{S}$ the graph $\left(S, E_{S}\right)$ whose edge relation is

$$
E_{S}=\{\langle v, w\rangle: v \in S \wedge w \in S \wedge w \in v\}
$$

and call MEMBERSHIP GRAPH of $S$ the pointed graph $\left(G_{\operatorname{TrCl}(\{S\})}, S\right)$.

Proposition 1.1. The membership graph of any hereditarily finite set in $\mathrm{HF}$ has the identity relation as its only bisimulation.

Proposition 1.1 has the following converse:

Proposition 1.2. Any finite, acyclic, pointed graph having identity as its only bisimulation is isomorphic to the membership graph of a hereditarily finite set in HF.

On the basis of the above two propositions, one can identify HF with the collection of those finite, acyclic, pointed graphs whose only bisimulation is the identitywhich, in its turn, is the collection of those finite acyclic pointed graphs in which no two different nodes have the same successor set. 
As said in the Introduction, our main task is to extend Ackermann's bijection $\mathbb{N}_{A}$, and ordering $\prec$, from $\mathrm{HF}$ to $\mathrm{HF}^{1 / 2}$; hence, in order to bring hypersets into play, we must generalize the graph-theoretical view of sets just introduced.

Definition 3. A HYPERSET is (the isomorphism class of) a pointed graph on which identity is the only bisimulation. Such an entity is said to be HEREDITARILY FINITE when it has finitely many nodes.

Recalling that the SUBGRAPH ISSUING FROM $w$ in a graph $G$ is the subgraph, pointed in $w$, that consists of all nodes which are reachable from $w$ in $G$, we can readily introduce the membership relation between hypersets as follows.

Definition 4. Given two hypersets $h$ and $h^{\prime}=(G, v)$, with $G=(V, E)$ as usual, we say that $h \in h^{\prime}$ if and only if $h$ is (isomorphic to) a pointed subgraph of $G$ issuing from a node $w$ with $\langle v, w\rangle \in E$.

In view of the natural embedding of the hereditarily finite sets, as classically understood, into the hereditarily finite hypersets just introduced, one says that an $a \in \mathrm{HF}^{1 / 2}$ is WELL-FOUNDED if and only if $a \in \mathrm{HF}$. It is NON-WELL-FOUNDED otherwise.

Turning now our attention to Ackermann's function $\mathbb{N}_{A}(\cdot)$, introduced above, from the hereditarily finite sets to natural numbers, its defining formula $\mathbb{N}_{A}(a)=$ $\Sigma_{b \in a} 2^{\mathbb{N}_{A}(b)}$ can be worded as follows:

(1) the binary representation of $\mathbb{N}_{A}(a)$ has a 1 in position $\mathbb{N}_{A}(b)$ if and only if $b \in a$.

Example 1. The Ackermann number of $\emptyset$ is 0 , which in fact shows that no set $b$ is element of $\emptyset$. The Ackermann number of $\{\{\{\emptyset\}\}\}$ is 4 , which in binary is 100 , namely the code of a set whose only element has code 2 (and hence is the set $\{\{\emptyset\}\}$ ). The Ackermann number of $\{\{\{\emptyset\}\}, \emptyset\}$ is 5 .

The above wording of $\mathbb{N}_{A}$ directly suggests its main properties. For example, to see the bijectivity of $\mathbb{N}_{A}$ it suffices to observe that

- a simple reading - from right to left - of the digits of any $n \in \mathbb{N}$ enables one to inductively determine the extension of the set $a$ such that $n=\mathbb{N}_{A}(a)$;

- a simple recursive routine manipulating sets and integers in binary representation can be devised so that it produces $n=\mathbb{N}_{A}(a)$ out of any given hereditarily finite set $a$.

As regards the order induced over the hereditarily finite sets by the Ackermann numbering, simple considerations allow us to prove that this ordering complies with rank comparison and is, actually, fully described by the anti-lexicographic criterion that

$$
a \prec b \Leftrightarrow \max _{\prec}(a \backslash b) \prec \max _{\prec}(b \backslash a)
$$

as recalled above.

Remark 1.3. We can easily check (cf. also [LS99]) that an extension of the Ackermann order to the entire $\mathrm{HF}^{1 / 2}$ cannot be carried out naively on the basis of the above property 2 . To see this, consider the hypersets $a=\{b\}, b=\{a, \emptyset\}$. Since $\emptyset \prec a$, we have $\max _{\prec}\{c: c \in a \backslash b\}=b$ and $\max _{\prec}\{c: c \in b \backslash a\}=a$. Property 2 then implies $a \prec b \Leftrightarrow b \prec a$ : a contradiction. 
A less naive attempt - which will, in fact, ultimately work - starts with the Ackermann function $\mathbb{N}_{A}$ (from which the Ackermann order can be defined), and tries to extend it to an encoding (i.e. $\mathbb{Q}_{A}$ ) from $\mathrm{HF}^{1 / 2}$ to a codomain (i.e. $\mathbb{Q}_{2}^{+}$) larger than $\mathbb{N}$. This must be done in such a way as to maintain the characteristic properties of $\mathbb{N}_{A}$, which we can state as follows:

- a "simple" recursive routine manipulating sets should allow one to get the code $y=\mathbb{Q}_{A}(a)$ from any given $a$;

- a "simple" reading of the code $y$ should allow one to inductively determine the extension of the (unique) set $a$, such that $y=\mathbb{Q}_{A}(a)$.

We would also like to have a property corresponding to 1 , which suggests what follows. As natural numbers have a twofold purpose in the Ackermann coding, being used both as positions inside a code $\mathbb{N}_{A}(a)$ and as the code itself, what happens if we split this purpose into two, by means of two functions, one assigning a position to each hereditarily finite hyperset and the other assigning a code to it? Since positions relative to natural numbers are already occupied by well-founded sets, it is natural to add negative positions to be used for non-well-founded sets. This will result in employing an extra function $\mathbb{Z}_{A}$ mapping into integers in place of natural numbers. By proceeding as outlined, we naturally obtain a (dyadic) rational number $\mathbb{Q}_{A}(a)$ as code of $a$, and we shall aim at the following extension of property 1:

the binary representation of $\mathbb{Q}_{A}(a)$ has a 1 in position $\mathbb{Z}_{A}(b)$ if and only if $b \in a$,

with both $\mathbb{Z}_{A}$ and $\mathbb{Q}_{A}$ having $\mathbb{N}_{A}$ as their restriction to HF.

In prospect of defining the function $\mathbb{Z}_{A}$, which will be obtained via an extension to $\mathrm{HF}^{1 / 2}$ of the Ackermann order $\prec$ on $\mathrm{HF}$, we now provide a concrete characterization of $\prec$, based on the so-called splitting technique (see also [LS99]).

\section{A New Look at the Ackermann Order}

We will work out in Sec. 2.1 an inductive characterization of Ackermann order $\prec$ of HF, grounding it on the splitting technique devised in [PT87], which was subsequently refined - to cite two among many - in [DPP04] and [PP04]. The splitting technique is an ingredient of an algorithm for computing bisimilarity on a graphe.g., for computing the equality relation on $\mathrm{HF}^{1 / 2}$. In the ongoing, it will be used to impose an order on HF; then, in Sec. 3, it will be used to order $\mathrm{HF}^{1 / 2}$ similarly.

One starts with a PARTITION, i.e., with a set consisting of pairwise disjoint nonnull sets, henceforth named BLOCKS of the partition. We write $\pi \sqsubseteq \pi^{\prime}$ (read " $\pi$ is finer than $\pi^{\prime \prime}$ " or " $\pi^{\prime}$ is coarser than $\pi^{\prime \prime) ~ t o ~ i n d i c a t e ~ t h a t ~} \pi$ and $\pi^{\prime}$ are partitions, with $\bigcup \pi=\bigcup \pi^{\prime}$, and every block of $\pi$ is included in a block of $\pi^{\prime}$.

When $\pi$ is a partition and $R$ is a binary relation on $\bigcup \pi$, denoting the preimage of a block $q$ by $R^{-1}[q]=_{\text {Def }}\{x: x \in \bigcup \pi \wedge \exists y \in q x R y\}$, we also define

$$
\pi \bumpeq R \Leftrightarrow \Leftrightarrow_{\text {Def }} \forall p \in \pi \forall q \in \pi\left(p \cap R^{-1}[q] \neq \emptyset \rightarrow p \subseteq R^{-1}[q]\right)
$$

(read " $\pi$ is $R$-stable"). Otherwise stated, stability requires that for all blocks $p$ and $q$, every element of $p$ is sent into $q$ via $R$, provided even a single element of $p$ is sent into $q$. Trivially, any partition whose blocks are singletons is stable. Another example of an $R$-stable partition is $\pi=\{\{a, b\},\{c, d\},\{e, f, g\}\}$, if $R=$ $\{(a, c),(b, c)\}$, while if we add the edge $(e, a)$ to $R$ the resulting partition is not 
stable. Intuitively, a partition is not $R$-stable if $R$ is able to "see" two elements belonging to one of its blocks as inequivalent.

Bisimulation problems can be stated as follows (see also [GPP03] for more details and an extension of this point of view to simulation): given a partition $\pi^{\star}$ along with a relation $R$ on $\bigcup \pi^{\star}$, find the coarsest $\pi_{\star}$ of all partitions that refine $\pi^{\star}$ and are $R$-stable. Formally:

$$
\forall \pi \sqsubseteq \pi^{\star}\left(\pi \bumpeq R \Leftrightarrow \pi \sqsubseteq \pi_{\star}\right) .
$$

Proceeding top-down, one can begin with $\pi=\pi^{\star}$ to then replace within $\pi$, as long as there are blocks $p, q$ for which $p \cap R^{-1}[q]$ and $p \backslash R^{-1}[q]$ are nonnull, $p$ by the latter two sets. If $\bigcup \pi^{\star}$ is finite, one will at last attain the desired $\pi_{\star}$ as value of $\pi$; more or less rapidly, depending on the order in which blocks are processed and split.

Within the stabilization process, the basic splitting action, namely replacing $p$ by $p \cap R^{-1}[q]$ and $p \backslash R^{-1}[q]$, can be packaged together with many other actions of the same kind. For example (as proposed in [PT87]), one can trace all $p$ 's which can be split by the same $q$, and replace each of them by the resulting two blocks before seeking another $q$. Proceeding the other way around (as we will do), one can locate a $p$ which is unstable relative to at least one $q$ and then supersede $p$ inside $\pi$, in a single shot, by all equivalence classes into which $p$ gets partitioned by the equivalence relation

$$
x \sim_{R} y \Leftrightarrow \Leftrightarrow_{\text {Def }} \forall q \in \pi\left(x \in R^{-1}[q] \Leftrightarrow y \in R^{-1}[q]\right) .
$$

In the two cases which we will study, $R$ will be $\ni$, while the initial partition $\pi^{\star}$ will first satisfy $\bigcup \pi^{\star}=\mathrm{HF}$ and then $\bigcup \pi^{\star}=\mathrm{HF}^{1 / 2}$. Despite $\bigcup \pi^{\star}$ being infinite in either case, infinite repetition of the basic splitting action will end into something valuable. To set the ground for this on a simple preliminary example, suppose here that $\pi^{\star}=\{\mathrm{HF}\}$, let $\pi_{0}=\pi^{\star}$, and then for $n=0,1,2, \ldots$ :

- observe that there is exactly one infinite block $p_{n} \in \pi_{n}$;

- observe that $p_{n}$ is a witness of the instability of $\pi_{n}$, as the sets

$$
\left\{x: x \in p_{n} \wedge x \cap p_{n} \neq \emptyset\right\} \text { and }\left\{x: x \in p_{n} \wedge x \cap p_{n}=\emptyset\right\}
$$

are nonnull (actually, the former is infinite);

- put

$$
\pi_{n+1}=\left(\pi_{n} \backslash\left\{p_{n}\right\}\right) \cup\left\{\left\{x: x \in p_{n} \wedge x \cap p_{n} \neq \emptyset\right\},\left\{x: x \in p_{n} \wedge x \cap p_{n}=\emptyset\right\}\right\},
$$

that is, we split the class $p_{n}$ by using $p_{n}$ itself as a splitter. At the conclusion, $\left\{\left\{x: x \in p_{n} \wedge x \cap p_{n}=\emptyset\right\}: n \in \mathbb{N}\right\}$ turns out to be the partition of HF whose blocks are the rank-equality classes. These blocks are all finite, but not singletons: an indication, since stable partitioning must give us the bisimilarity classes, that stability has not been attained as yet.

In what we are about to see, we resume work with the partition just found. We will sequence successive splitting actions fairly enough that the stable partition will result after denumerably many actions; along the way, we will impose an order on the singleton blocks.

2.1. An inductive definition of the Ackermann Order. Processing the collection $\mathrm{HF}$ will amount to defining a countable sequence $\left(\mathcal{X}^{n}\right)_{n \in \mathbb{N}}$ of ordered partitions $\mathcal{X}^{n}=\left\{X_{i}^{n}: i \in \mathbb{N}\right\}$ of it. Each partition $\mathcal{X}^{n+1}$ will turn out to be an ORDERED 
REFINEMENT of $\mathcal{X}^{n}$, namely (for all $i, j, h, k \in \mathbb{N}$ ):

$$
\begin{gathered}
\exists k\left(X_{i}^{n+1} \subseteq X_{k}^{n}\right), \\
X_{i}^{n+1} \subseteq X_{k}^{n} \wedge X_{j}^{n+1} \subseteq X_{h}^{n} \wedge k>h \Rightarrow i>j .
\end{gathered}
$$

That is, $\mathcal{X}^{n+1} \sqsubseteq \mathcal{X}^{n}$ and the ordering of the subblocks into which the blocks of $\mathcal{X}^{n}$ get split in the formation of $\mathcal{X}^{n+1}$ will be consistent with the preceding ordering.

For all $n$, we will maintain the invariant:

$$
\text { Is_finite }\left(X_{i}^{n}\right) \wedge\left(x \in X_{h}^{n} \wedge \operatorname{rk}(y)<\operatorname{rk}(x) \wedge y \in X_{k}^{n} \Rightarrow h>k\right),
$$

implying that the blocks of $\mathcal{X}^{n}$ are finite and they, as well as their elements, are ordered in a way complying with rank comparison-hence complying, in this wellfounded case, with membership ${ }^{8}$. This is important because we want sets to be sorted à la Ackermann when, at the end of the process, the partition will be $\ni-$ stable and blocks will be singletons. To meet 6 at the outset, we define $\mathcal{X}^{0}$ by putting

$$
X_{i}^{0}=\{x: x \in \mathrm{HF} \wedge \mathrm{rk}(x)=i\} \quad \text { for all } i \in \mathbb{N} .
$$

Preliminary to defining $\mathcal{X}^{n+1}$, we consider the smallest index $h$ such that the block $X_{h}^{n}$ can be split in the sense that there exist $x, y \in X_{h}^{n}$, and some $k$, such that $x$ shares elements with $X_{k}^{n}$ whereas $y$ does not. We also consider the equivalence relation $\sim_{\ni}$ on $X_{h}^{n}$ given by

$$
x \sim_{\ni} y \Leftrightarrow \forall k\left(X_{k}^{n} \cap x=\emptyset \leftrightarrow X_{k}^{n} \cap y=\emptyset\right) .
$$

Then we consider the partition induced by $\sim_{\ni}$ on $X_{h}^{n}$, ordered as follows: given two $\sim_{\ni}$-classes $Z^{\prime}, Z \subseteq X_{h}^{n}$, put $Z^{\prime}$ before $Z$ if and only if, for $w \in Z^{\prime}$ and $z \in Z$, the largest mismatch position $k$ between $w, z$ 'favors' $z$, i.e.

$$
X_{k}^{n} \cap w=\emptyset \wedge X_{k}^{n} \cap z \neq \emptyset \wedge \forall j>k\left(X_{j}^{n} \cap w=\emptyset \leftrightarrow X_{j}^{n} \cap z=\emptyset\right) .
$$

It plainly ensues from the definition of $\sim_{\ni}$ that the mismatch position does not depend on the choice of $w$ and $z$; hence this relationship imposes an order $Z_{0}, Z_{1}, \ldots, Z_{m}$ $(m \geqslant 1)$ on the $\sim_{\ni}$-equivalence classes of $X_{h}^{n}$. On this ground we can put:

$$
X_{i}^{n+1}= \begin{cases}X_{i}^{n} & \text { if } i<h, \\ Z_{i-h} & \text { if } h \leqslant i \leqslant h+m, \\ X_{i-m}^{n} & \text { if } h+m<i .\end{cases}
$$

In the well-founded case at hand, an inductive argument on $n$ shows that the smallest index $h$ such that $X_{h}^{n}$ can be split coincides with the smallest index $h$ such that $X_{h}^{n}$ is not a singleton; moreover, it turns out that the relation $\sim_{\ni}$ induces a partition of $X_{h}^{n}$ into singleton blocks. These verifications are straightforward, and we leave them to the reader.

Properties 6, 4, and 5 hold throughout the construction and every element of HF will eventually belong to a singleton class. Given $n \in \mathbb{N}$ and $x \in \mathrm{HF}$, let $f(x, n) \in \mathbb{N}$ be such that

$$
x \in X_{f(x, n)}^{n} .
$$

\footnotetext{
${ }^{8}$ Notice that, since our initial partition will separate sets at different ranks, we are guaranteed to have $h \neq k$ in the second conjunct of 6 .
} 
Then one can easily prove that the full Ackermann order of Sec. 1.2 is the limit of the $\mathcal{X}^{n}$ 's, that is:

$$
x \prec y \Leftrightarrow \exists n(f(x, n)<f(y, n)) .
$$

The previous construction will be generalized next to non-well-founded sets, by producing a sequence $\left(\mathcal{Y}^{n}\right)_{n \in \mathbb{N}}$ of ordered partitions, whose limit linearly orders $\overline{\mathrm{HF}}$. For all $n \in \mathbb{N}$, the ordered partition $\mathcal{Y}^{n+1}$ will still be an ordered refinement of $\mathcal{Y}^{n}$, but we will not have the possibility to prove that $\mathcal{Y}^{n+1}$ results from splitting into singleton classes the first class of $\mathcal{Y}^{n}$ which is not a singleton: in spite of the close analogy between the constructions, the splitting process will behave differently in the non-well-founded case.

\section{THE ORDER ON NON-WELL-FOUNDED HEREDITARILY FINITE SETS}

Let us say that a linear order $\prec$ is an ACKERMANN ORDER if it extends the Ackermann order of HF to a superset of HF. In order to get such an order on $\mathrm{HF}^{1 / 2}$, we will mimick the splitting process just given for HF. In analogy with the above, we will build a sequence $\left(\mathcal{Y}^{n}\right)_{n \in \mathbb{N}}$ of ordered partitions $\mathcal{Y}^{n}=\left\{Y_{i}^{n}: i \in \mathbb{N}\right\}$ of $\mathrm{HF}^{1 / 2}$, where each partition $\mathcal{Y}^{n+1}$ is an ordered refinement of $\mathcal{Y}^{n}$. The $\mathcal{Y}^{n}$ 's are constructed inductively again, starting with an $\mathcal{Y}^{0}$ which, by way of first approximation, is taken arbitrarily; as we will see, a linear order $\prec$ on $\mathrm{HF}^{1 / 2}$ will result as the limit of the sequence $\left(\mathcal{Y}^{n}\right)_{n \in \mathbb{N}}$ if all blocks in $\mathcal{Y}^{0}$ are finite. One further restraint will be imposed at the outset in order that $\prec$ be an Ackermann order on $\mathrm{HF}^{1 / 2}$ : we will require that the initial $\mathcal{Y}^{0}$ be coherent with the (standard) Ackermann order on HF.

3.1. The splitting procedure on $\mathrm{HF}^{1 / 2}$. At step $n+1$, the ordered partition $\mathcal{Y}^{n+1}$ is defined as a refinement of $\mathcal{Y}^{n}$, in complete analogy with the splitting action exploited in the well-founded case. We say that a block $Y_{i}^{n}$ can be split if it contains two inequivalent elements with respect to the relation $\sim_{\ni}$ defined (as above) by

$$
x \sim \ni \quad \Leftrightarrow \quad \forall j\left(Y_{j}^{n} \cap x=\emptyset \leftrightarrow Y_{j}^{n} \cap y=\emptyset\right) .
$$

By considering the smallest number $h$ such that $Y_{h}^{n}$ can be split, and the partition of the block $Y_{h}^{n}$ induced by $\sim_{\ni}$, we proceed exactly as before to sort the $\sim_{\ni}$ equivalence classes of $Y_{h}^{n}$ as $Z_{0}, Z_{1}, \ldots, Z_{m}(m \geqslant 1)$. Then we put:

$$
Y_{i}^{n+1}= \begin{cases}Y_{i}^{n} & \text { if } i<h, \\ Z_{i-h} & \text { if } h \leqslant i \leqslant h+m \\ Y_{i-m}^{n} & \text { if } h+m<i\end{cases}
$$

In sight of getting a linear order of $\mathrm{HF}^{1 / 2}$, we define as before the dyadic relation

$$
x \prec y \Leftrightarrow \exists n(f(x, n)<f(y, n))
$$

over $\mathrm{HF}^{1 / 2}$ in terms of the function $f: \mathrm{HF}^{1 / 2} \times \mathbb{N} \longrightarrow \mathbb{N}$ such that $x \in Y_{f(x, n)}^{n}$.

However, as we see in the following example, the relation $\prec$ is not necessarily a linear order, unless we impose some further conditions on the initial partition $\mathcal{Y}^{0}$.

Example 2. Suppose $\mathcal{Y}^{0}=\left\{\mathrm{HF}^{1 / 2}\right\}, x=\Omega$, and $y=\{\emptyset, \Omega\}$ for the unique hyperset $\Omega$ such that $\Omega=\{\Omega\}$. Then $f(x, 2)<f(y, 2)$ and hence $x \prec y$. As is easily proved by induction, for all $n$ the class $Y_{f(x, n)}^{n}$ contains, besides $x$, the sequence $\{\emptyset\}^{n},\{\emptyset\}^{n+1},\{\emptyset\}^{n+2}, \ldots$ where $\{\emptyset\}^{0}=\emptyset$ and $\{\emptyset\}^{n+1}=\left\{\{\emptyset\}^{n}\right\}$. It follows that $f(x, n)$ coincides with the smallest index $h$ such that $Y_{h}^{n}$ can be split. This implies 
that the non-singleton class $Y_{f(y, n)}^{n}$ is never split, and if $z \in Y_{f(y, n)}^{n} \backslash\{y\}$ then neither $z \prec y$ nor $y \prec z$ holds.

We next give a necessary and sufficient condition for the relation $\prec$ defined in 10 to be a linear order on $\mathrm{HF}^{1 / 2}$ :

Lemma 3.1. The relation $\prec$ is a linear order if and only if

(11) $\forall x, y \in \mathrm{HF}^{1 / 2}\left(\forall n(f(x, n)=f(y, n)) \rightarrow \forall n \forall j\left(Y_{j}^{n} \cap x=\emptyset \leftrightarrow Y_{j}^{n} \cap y=\emptyset\right)\right)$,

(i.e., iff any sets $x, y$ in $\mathrm{HF}^{1 / 2}$ that remain forever together in the same block never mismatch).

Proof. Condition 11 is clearly necessary in order that $\prec$ be a linear order.

Conversely, suppose 11 holds. Preliminary to proving that $\prec$ is a linear order, observe that $\prec$ is irreflexive and transitive; hence we must only prove that when $x \neq y$ holds there exists $n \in \mathbb{N}$ such that $f(x, n) \neq f(y, n)$. This in turn follows from the fact that the relation $b \subseteq \mathrm{HF}^{1 / 2} \times \mathrm{HF}^{1 / 2}$ defined by

$$
x b y \Leftrightarrow \forall n(f(x, n)=f(y, n))
$$

is a bisimulation. To see this, suppose $x$ b $y$ and $x^{\prime} \in x$; then $x^{\prime} \in Y_{f\left(x^{\prime}, n\right)}^{n} \cap x$ for all $n \in \mathbb{N}$. By 11 we obtain that also $Y_{f\left(x^{\prime}, n\right)}^{n} \cap y \neq \emptyset$ for all $n \in \mathbb{N}$. Since $y$ is a finite set, from $Y_{f\left(x^{\prime}, n\right)}^{n} \cap y \neq \emptyset$ for all $n \in \mathbb{N}$ we deduce the existence of an element $y^{\prime} \in y$ belonging to all classes $Y_{f\left(x^{\prime}, n\right)}^{n}$. This implies that $x^{\prime} b y^{\prime}$.

Likewise, $x$ b $y$ and $y^{\prime} \in y$ implies the existence of an $x^{\prime} \in x$ such that $x^{\prime} b y^{\prime}$.

One simple (and natural) choice to achieve condition 11 of Lemma 3.1 is to start the splitting process from a partition composed of finite sets, as the following Corollary shows.

Corollary 3.2. If $\mathcal{Y}^{0}=\left\{Y_{i}^{0}: i \in \mathbb{N}\right\}$, where every $Y_{i}^{0}$ is finite, then $\prec$ linearly orders $\mathrm{HF}^{1 / 2}$.

Proof. Using Lemma 3.1 we can prove that $\prec$ is a linear order by proving that 11 holds. Assume $x$ and $y$ are such that there exists a stage $n$ and a position $j$ such that $Y_{j}^{n} \cap x=\emptyset$ and $Y_{j}^{n} \cap y \neq \emptyset$. If $x$ and $y$ belong to the same class $Y_{i}^{n}$, it follows from our hypothesis on $\mathcal{Y}^{0}$ that at stage $n$ the number of elements belonging to classes preceding $Y_{i}^{n}$ is finite. This is sufficient to guarantee that $x$ and $y$ will be eventually separated.

Corollary 3.2 ensures the existence of infinitely many linear orders on $\mathrm{HF}^{1 / 2}$ built up using the splitting procedure. Among them, as we will show below, we find an Ackermann order when the first partition $\mathcal{Y}^{0}$ is defined by resorting to a suitable notion of rank.

3.2. A rank notion for $\mathrm{HF}^{1 / 2}$. In order to be able to apply Corollary 3.2, we now want to define a suitable notion of rank for the hereditarily finite hypersets $\mathrm{HF}^{1 / 2}$. Our definition will have the following features: on the one hand, when specialised to the case of well-founded sets it will coincide with the standard notion given in Sec. 1 , on the other hand, it will guarantee that only a computable number of hypersets will have a given finite rank.

In the well-founded case, the notion of rank can be seen to correspond to the length of the longest simple path issuing from $x$ in $G_{\operatorname{TrCl}(\{x\})}$. The following example 
shows that if we put a bound on the length of the longest simple path from $x$ and require that no two isomorphic pointed subgraphs are present in $\left(G_{\operatorname{TrCl}(\{x\})}, x\right)$, we can have graphs with arbitrarily many nodes.

Example 3. Consider the graph in Figure 2 for arbitrarily large $n$, where the set of nodes is $\left\{x, b_{1}, \ldots, b_{n}, c_{1}, \ldots, c_{n}\right\}$, and the edge relation is

$$
\left\{\left(x, b_{i}\right),\left(c_{i}, x\right): i \geqslant 1\right\} \cup\left\{\left(b_{i}, c_{j}\right): j \geqslant i \geqslant 1\right\} .
$$

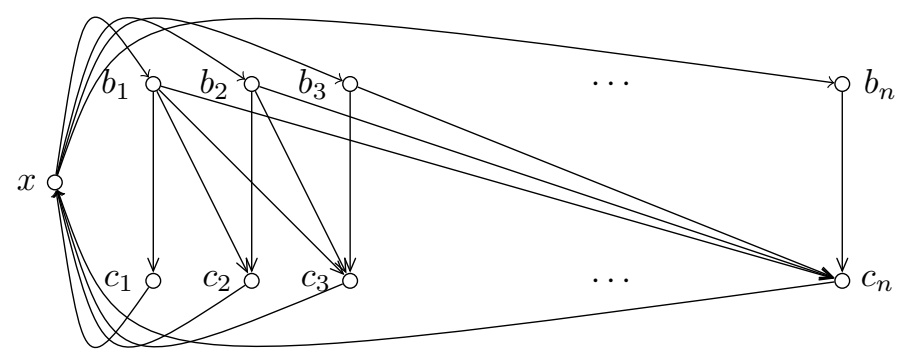

Figure 2. A template to build pointed graphs with arbitrarily many nodes, all simple paths starting in their point of length less than or equal to two, and no isomorphic pointed subgraphs.

The length of the longest simple path from $x$ in the graph below is two. Moreover, as one can easily see, no pairwise distinct nodes $y$ and $z$ are such that $\left(G_{\operatorname{TrCl}(\{y\})}, y\right)$

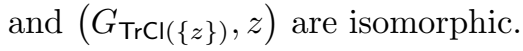

The above example suggests that, if we want to limit the number of membership graphs that we are able to build at any given rank, the notion of rank must not only limit paths in their lengths, but must also put a limit on the number of outgoing arcs from any given node. This is automatically achieved if we put a bound on path lengths and consider only graphs corresponding to transitive closure of well-founded sets (as we shall see in Lemma 3.4, in these graphs the maximal length of a simple path also limits each node degree). When dealing with sets in $\mathrm{HF}^{1 / 2}$ we have to impose a limit on both path lengths and node degrees.

Given $x \in \mathrm{HF}^{1 / 2}$ and $y \in \operatorname{TrCl}(\{x\})$ we denote by $\bar{d}(x, y)$ the length of the longest

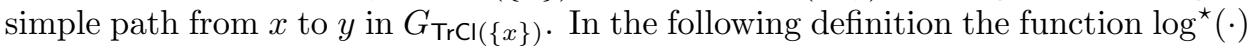
stands for the iterated (binary) logarithm.

Definition 5. For any $x \in \mathrm{HF}^{1 / 2}$, the RANK $\mathrm{rk}(x)$ of $x$ is

$$
\max \left\{\bar{d}(x, y), \log ^{\star}(|y|): y \in \operatorname{TrCl}(\{x\})\right\} .
$$

As we shall see in Lemma 3.4, the use of the iterated logarithm in the previous definition is instrumental to obtaining a notion of rank which is generalization of the usual definition in HF.

Lemma 3.3. There exists a computable function $f$ such that

$$
\left|\left\{x \in \mathrm{HF}^{1 / 2} \mid \operatorname{rk}(x) \leqslant n\right\}\right| \leqslant f(n) .
$$


Proof. Given $x \in \mathrm{HF}^{1 / 2}$ such that $\mathrm{rk}(x)=n$, consider $G_{\operatorname{TrCl}(\{x\})}$. By definition of rank we know that the length of the longest simple path in $G_{\operatorname{TrCl}(\{x\})}$ is less than $n$. Moreover, again by definition of rank, every $y \in \operatorname{TrCl}(\{x\})$ is such that $\log ^{\star}(|y|) \leqslant n$, and therefore the number of outgoing arcs from $y$ must be less than or equal to $k=\beth(n)$, where

$$
\beth(i)= \begin{cases}0 & \text { if } i=0, \\ 2^{\beth(i-1)} & \text { if } i>0 .\end{cases}
$$

To conclude, recall that all the nodes in $G_{\operatorname{TrCl}(\{x\})}$ are reachable from $x$. Hence, by induction on the distance from $x$, it can be easily proved that the nodes in $G_{\operatorname{TrCl}(\{x\})}$ are at most the nodes in a complete $k$-ary tree of height $n$. That is $k^{n}$.

Lemma 3.4. For all $x \in \mathrm{HF}$, we have that

$$
\max \left\{\bar{d}(x, y), \log ^{\star}(|y|): y \in \operatorname{TrCl}(\{x\})\right\}=\sup \{\operatorname{rk}(y)+1: y \in x\} .
$$

Proof. To see that whenever $x$ is well-founded the above equality holds, observe that $\sup \{\operatorname{rk}(y)+1: y \in x\}=\bar{d}(x, \emptyset)=\max \{\bar{d}(x, y): y \in \operatorname{TrCl}(\{x\})\}$. Let $\bar{d}(x, \emptyset)=n$. Since the maximum cardinality of a well-founded set of rank $k$ is $\beth(k)$, for every $y \in \operatorname{TrCl}(\{x\})$, we have that $\log ^{\star}(|y|) \leqslant \log ^{\star}(\beth(n))=n$. Therefore:

$$
n=\max \{\bar{d}(x, y): y \in \operatorname{TrCl}(\{x\})\}=\max \left\{\bar{d}(x, y), \log ^{\star}(|y|): y \in \operatorname{TrCl}(\{x\})\right\} .
$$

\subsection{An Ackermann Order on $\mathrm{HF}^{1 / 2}$. We start with the partition}

$$
\mathcal{Y}^{0}=\left\{Y_{i}^{0}: i \in \mathbb{N}\right\},
$$

where $Y_{i}^{0}=\left\{x: x \in \mathrm{HF}^{1 / 2} \wedge \mathrm{rk}(x)=i\right\}$, for all $i \geqslant 0$. Consider the splitting sequence $\left(\mathcal{Y}^{n}\right)_{n \in \mathbb{N}}$ defined as in Sec. 3.1. By Lemma 3.3, each class $Y_{i}^{0}$ contains a finite number of hypersets, and from Corollary 3.2 it follows that the order $\prec$, defined by

$$
x \prec y \Leftrightarrow \exists n \in \mathbb{N}(f(x, n)<f(y, n)),
$$

is a linear order on $\mathrm{HF}^{1 / 2}$. Since the construction is a generalization of the splitting procedure on $\mathrm{HF}$, and well-founded sets only contain well-founded sets, the order $\prec$ extends the Ackermann order on HF.

Example 4. We consider as an example the $\mathrm{HF}^{1 / 2}$ sets depicted in Fig. 3.

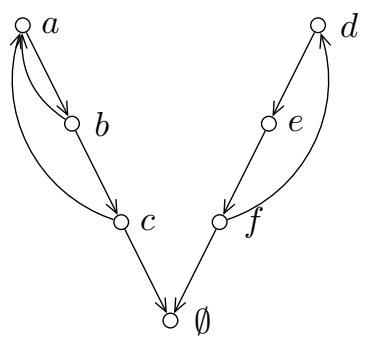

Figure 3. Hypersets $a=\{b\}, b=\{c, a\}, c=\{a, \emptyset\}, d=$ $\{e\}, e=\{f\}, f=\{d, \emptyset\}$ 
The sets $a, b, c, d, e, f$ are non-well-founded; $a, d$ have rank equal to 3 , while $b, c, e, f$ have rank equal to 2 . Hence $a, d$ belong to $Y_{3}^{0}$, while $b, c, e, f$ belong to $Y_{2}^{0}$. The the splitting procedure goes as follows:

$$
\begin{gathered}
{[\emptyset, \ldots] \ldots[b, c, e, f \ldots][a, d \ldots] \ldots} \\
{[\emptyset] \ldots[e \ldots][c, f \ldots][b \ldots][a, d \ldots] \ldots} \\
{[\emptyset] \ldots[e \ldots][c, f \ldots][b \ldots][d \ldots][a \ldots] \ldots} \\
{[\emptyset] \ldots[e \ldots][f \ldots][c \ldots][b \ldots][d \ldots][a \ldots] \ldots}
\end{gathered}
$$

Hence, the final order $\prec$ on $a, b, c, d, e, f$ satisfies

$$
\emptyset \prec e \prec f \prec c \prec b \prec d \prec a
$$

Remark 3.5. Notice that the extended Ackermann order $\prec$ resulting from the above construction is by no means unique. Arguing as in the preceding section, in fact, we see that the splitting process could have started with any partition $\mathcal{Y}^{0}=\left\{Y_{i}^{0}: i \in\right.$ $\mathbb{N}\}$ composed of finite sets $Y_{i}^{0}$ with $Y_{i}^{0} \supseteq\{x: x \in \mathrm{HF} \wedge \operatorname{rk}(x)=i\}$, e.g. by using a notion of rank for $\mathrm{HF}^{1 / 2}$ where, instead of the iterated logarithm, a slower function is used: the limit of the sequence $\left(\mathcal{Y}^{n}\right)_{n \in \mathbb{N}}$ would then have been an Ackermann order as well.

The above remark suggests that in the presence of non-well-founded sets, different notions of rank can be used to ground the splitting procedure.

\section{HeREditarily Finite HyPERSETS AS DYADIC NUMBER}

We are now ready to introduce the extension of the Ackermann function. First, we use the order $\prec$ on $\mathrm{HF}^{1 / 2}$ defined in Sec. 3.3 to give positions to sets in $\mathrm{HF}^{1 / 2}$. As explained before, we need to use integer positions, since natural positions are already occupied by well-founded sets.

If $a \in \mathrm{HF}^{1 / 2}$, define:

$$
\mathbb{Z}_{A}(a)=\left\{\begin{array}{cl}
|\{b: b \in \mathrm{HF} \wedge b \prec a\}| & \text { if } a \in \mathrm{HF}, \\
-\left|\left\{b: b \in \mathrm{HF}^{1 / 2} \backslash \mathrm{HF} \wedge b \prec a\right\}\right|-1 & \text { if } a \in \mathrm{HF}^{1 / 2} \backslash \mathrm{HF} .
\end{array}\right.
$$

Let $\mathbb{Q}_{2}^{+}$be the set of all dyadic numbers, that is,

$$
\mathbb{Q}_{2}^{+}=\left\{\frac{n}{2^{m}}: n, m \in \mathbb{N}\right\} \text {. }
$$

Dyadic numbers are rational numbers having a binary expansion with a finite number of digits.

We define a bijection $\mathbb{Q}_{A}$ from $\mathrm{HF}^{1 / 2}$ to dyadic numbers as follows:

$$
\mathbb{Q}_{A}(a)=\Sigma_{b \in a} 2^{\mathbb{Z}_{A}(b)},
$$

All properties announced in Sec. 1.2 are satisfied. In particular:

- $\mathbb{Q}_{A}: \mathrm{HF}^{1 / 2} \rightarrow \mathbb{Q}_{2}^{+}$extends the Ackermann function $\mathbb{N}_{A}: \mathrm{HF} \rightarrow \mathbb{N}$; that is, $\mathbb{Q}_{A}(x)=\mathbb{N}_{A}(x)$ holds when $x \in \mathrm{HF}$.

- A simple reading of the code $y \in \mathbb{Q}_{2}^{+}$allows us to inductively determine the extension of the set $a$ such that $y=\mathbb{Q}_{A}(a)$. This is because from the digits of $y$ we determine the positions $\mathbb{Z}_{A}(b)$ of all $b \in a$, and, since the bijection $\mathbb{Z}_{A}$ is effective, from $\mathbb{Z}_{A}(b)$ we are able to determine $b$.

- A simple recursive routine manipulating sets allows us to build the code $y=\mathbb{Q}_{A}(a)$ from any given hereditarily finite set $a$, because if we know $a$ we can compute $\mathbb{Z}_{A}(b)$ for all $b \in a$, and hence $\mathbb{Q}_{A}(a)$. 
The twofold role of the Ackermann function $\mathbb{N}_{A}: \mathrm{HF} \rightarrow \mathbb{N}$, by which $\mathbb{N}_{A}(a)$ acts at the same time as code and as position for the hereditarily finite set $a$, must be played by distinct functions in the case of hypersets: for these, $\mathbb{Z}_{A}: \mathrm{HF}^{1 / 2} \rightarrow \mathbb{Z}$ defines positions while $\mathbb{Q}_{A}: \mathrm{HF}^{1 / 2} \rightarrow \mathbb{Q}_{2}^{+}$assigns codes.

4.1. Mapping $\mathbb{Q}_{2}^{+}$into $\mathrm{HF}^{1 / 2}$. A final, natural, question can arise when considering the mapping $\mathbb{Q}_{A}$ : is an inverse mapping - that is a mapping from $\mathbb{Q}_{2}^{+}$into $\mathrm{HF}^{1 / 2}$ _ definable?

Clearly, if no constraint is imposed, the answer is yes. However, if one requires some level of similarity with von Neumann's injection of $\mathbb{N}$ into HF, then the question becomes delicate. In our opinion, a minimal requirement to impose on any $h: \mathbb{Q}_{2}^{+} \rightarrow \mathrm{HF}^{1 / 2}$ extending bijectively von Neumann's one, is the following:

$$
\forall a \in \mathbb{Q}_{2}^{+} \forall x \in h(a) \exists b \in \mathbb{Q}_{2}^{+}(b \leqslant a \wedge x=h(b)) .
$$

It can be shown that a function $h$ satisfying the above property cannot be defined.

In fact, proceeding by contradiction, consider $a \in(0,1) \cap \mathbb{Q}_{2}^{+}$such that its transitive closure has minimal cardinality. By 12 it must be that $h(a)=\boldsymbol{\Omega}$ or - in case $h^{-1}(\boldsymbol{\Omega}) \geqslant 1$ - the solution of the equation $X=\{X, \emptyset\}$. Assume $h(a)=\boldsymbol{\Omega}$, the other case being treated analogously.

Let $b \in(0, a) \cap \mathbb{Q}_{2}^{+}$such that $h(b) \in h(a)$ and has transitive closure of minimal cardinality. Again by $12, h(b)$ must be the solution of the equation $X=\{X, \emptyset\}$. Then let $c \in(0, b) \cap \mathbb{Q}_{2}^{+}$. At this point notice that the only elements of $h(c)$ can be $h(c)$ or $\emptyset$. Therefore $h(c)$ can be either $\{\emptyset\}$, the solution $X=\{X, \emptyset\}$, or $\boldsymbol{\Omega}$. The first case cannot apply as $c$ would be equal to 1 . In the second case it would be $h(c)=h(b)$ and in the third case $h(c)=h(a)$, contradicting injectivity.

\section{A REAL-VALUED MAP}

Consider the following definition, obtained from the definition of $\mathbb{N}_{A}$ by simply adding a minus sign at exponent.

\section{Definition 6.}

$$
\mathbb{R}_{A}(x)=\Sigma_{y \in x} 2^{-\mathbb{R}_{A}(y)}
$$

The above definition bears a strong formal similarity with $\mathbb{N}_{A}$ but calls into play real numbers. This allows us to prove the existence of a unique solution to the following equation:

$$
x=2^{-x},
$$

putting us in a condition to view $\mathbb{R}_{A}$ as a map from hypersets into (real) numbers. To see the existence and unicity of a solution for 13, it suffices to observe that the two curves $y=x$ and $y=2^{-x}$ are increasing and decreasing, respectively, and they intersect only in the first quadrant of $\mathbb{R}^{2}$. Letting $\Omega$ be the solution of 13 over $\mathbb{R}$, the following holds.

\section{Proposition 5.1. $\Omega \notin \mathbb{Q}$}

Proof. Proceeding by contradiction, let $m / n=2^{-m / n}$. Plainly, $m \neq 0$ and $m<n$ (since $2^{-m / n}<1$ ). Assume $m$ and $n$ have no common divisors and, therefore, cannot be both even. Consider first the case in which $n=2 h+1$. In this case we would have that $(2 h+1) / m=2^{m / n}$ and therefore $(2 h+1)^{n}=m^{n} 2^{m}$, which is impossible, for the left-hand side is odd while the right-hand side is even. 
Assume then that $n$ is even and $m$ is odd. Putting $n=2 k$ and $m=2 h+1$, from $2^{m / n}=n / m$ we have

$$
2 k=2^{m / n}(2 h+1) \Leftrightarrow(2 k)^{n}=2^{m}(2 h+1)^{n} \Leftrightarrow 2^{n-m} k^{n}=(2 h+1)^{n},
$$

which is again a contradiction, since $m<n$ implies the left-hand side is even while the right-hand side is odd.

Our first objective should be to show that $\mathbb{R}_{A}$ is injective on HF.

Conjecture 5.2. The function $\mathbb{R}_{A}$ is injective on $\mathrm{HF}$.

A second, more challenging, result would consist in establishing the fact that $\mathbb{R}_{A}$ is injective on the full collection of rational hereditarily finite hypersets.

Conjecture 5.3. The function $\mathbb{R}_{A}$ is injective on $\mathrm{HF}^{1 / 2}$.

To justify our conjecture we determine the code of the hyperset satisfying the set-theoretic equation

$$
x=\{a, x\},
$$

for some fixed $a \in \mathrm{HF}^{1 / 2}$. The above $x$ has code $\mathbb{R}_{A}(x)$ satisfying

$$
\mathbb{R}_{A}(x)=2^{-\mathbb{R}_{A}(a)}+2^{-\mathbb{R}_{A}(x)},
$$

whose value we want to determine as the limit of the sequence of codes of sets

$$
x_{0}=\{a\}, x_{1}=\left\{a, x_{0}\right\}, x_{2}=\left\{a, x_{1}\right\}, \ldots, x_{i+1}=\left\{a, x_{i}\right\}, \ldots
$$

The codes of the above sets are

$\mathbb{R}_{A}\left(x_{0}\right)=2^{-\mathbb{R}_{A}(a)}, \mathbb{R}_{A}\left(x_{1}\right)=2^{-\mathbb{R}_{A}(a)}+2^{-\mathbb{R}_{A}\left(x_{0}\right)}, \ldots, \mathbb{R}_{A}\left(x_{i+1}\right)=2^{-\mathbb{R}_{A}(a)}+2^{-\mathbb{R}_{A}\left(x_{i}\right)}, \ldots$

Let us begin by observing that for any $i, j \in \mathbb{N}$ we have that:

(1) $\mathbb{R}_{A}\left(x_{2 i}\right)<\mathbb{R}_{A}\left(x_{2 i+2}\right)$, and $\mathbb{R}_{A}\left(x_{2 i+1)}>\mathbb{R}_{A}\left(x_{2 i+3}\right)\right.$,

(2) $\mathbb{R}_{A}\left(x_{2 i}\right)<\mathbb{R}_{A}\left(x_{2 j+1}\right)$.

(1) is easily seen to hold, inductively. For the base case notice that that $\mathbb{R}_{A}\left(x_{0}\right)<$ $\mathbb{R}_{A}\left(x_{2}\right)=\mathbb{R}_{A}\left(x_{0}\right)+2^{-\mathbb{R}_{A}\left(x_{1}\right)}$. The inductive step, in case the index is even follows from the inductive hypothesis relative to the odd case, and viceversa. In fact, $\mathbb{R}_{A}\left(x_{2 i}\right)<\mathbb{R}_{A}\left(x_{2 i+2}\right)$ is equivalent to

$$
2^{-\mathbb{R}_{A}(a)}+2^{-\mathbb{R}_{A}\left(x_{2 i-1}\right)}<2^{-\mathbb{R}_{A}(a)}+2^{-\mathbb{R}_{A}\left(x_{2 i+1}\right)},
$$

which is equivalent to $\mathbb{R}_{A}\left(x_{2 i-1}\right)>\mathbb{R}_{A}\left(x_{2 i+1}\right)$, that holds by the inductive hypothesis. The odd case is similar.

(2) is proved by first proving, that $\mathbb{R}_{A}\left(x_{2 i}\right)<\mathbb{R}_{A}\left(x_{2 i+1}\right)$, which is seen by induction. The base case consists in observing that $\mathbb{R}_{A}\left(x_{0}\right)<\mathbb{R}_{A}\left(x_{1}\right)=\mathbb{R}_{A}\left(x_{0}\right)+$ $2^{-\mathbb{R}_{A}\left(x_{0}\right)}$ holds, while the inductive case $\mathbb{R}_{A}\left(x_{2 i}\right)<\mathbb{R}_{A}\left(x_{2 i+1}\right)$ holds if and only if $2^{-\mathbb{R}_{A}\left(x_{2 i-1}\right)}<2^{-\mathbb{R}_{A}\left(x_{2 i}\right)}$, that follows from the inductive hypothesis. To conclude the proof of (2), assume that $i \leqslant j$ and observe that, applying (1) and the result just proved, $\mathbb{R}_{A}\left(x_{2 i}\right) \leqslant \mathbb{R}_{A}\left(x_{2 j}\right)<\mathbb{R}_{A}\left(x_{2 j+1}\right)$. In case $i \geqslant j$ the results follows analogously since $\mathbb{R}_{A}\left(x_{2 i}\right)<\mathbb{R}_{A}\left(x_{2 i+1}\right) \leqslant \mathbb{R}_{A}\left(x_{2 j+1}\right)$.

On the grounds of (1) and (2) we can conclude that the even indexed $\mathbb{R}_{A}\left(x_{i}\right)$ 's form an increasing sequence of reals lying to the left of the odd indexed ones that, in turn, constitute a decreasing sequence.

We now prove that both even and odd indexed $\mathbb{R}_{A}\left(x_{i}\right)$ converge to a value $r$ that will be then proved to be $\mathbb{R}_{A}(x)$. 
We begin by proving that $\left(2^{-x}-2^{-y}\right)<(y-x) / 2$, for all $x, y \in[1 / 2, \infty)$. This result follows from Lagrange theorem, stating that $(f(b)-f(a)) /(b-a)=f^{\prime}(z)$, for some $z \in(a, b)$. In fact, assuming $y>x$, we have

$$
\left(2^{-x}-2^{-y}\right)<\frac{y-x}{2} \Leftrightarrow \frac{2^{-x}-2^{-y}}{y-x}<\frac{1}{2} \Leftrightarrow-\frac{2^{-y}-2^{-x}}{y-x}<\frac{1}{2},
$$

that is , for some $z \in(x, y) \subseteq[1 / 2, \infty)$,

$$
-\left(2^{-z}\right)^{\prime}=2^{-z} \ln (2)<\frac{1}{2}
$$

which is always true for $z \geqslant 1 / 2$.

On the grounds of the above result we can prove that $\mathbb{R}_{A}\left(x_{2 i+1}\right)-\mathbb{R}_{A}\left(x_{2 i}\right)$ goes to 0 as $i$ goes to infinity. In fact, by definition,

$$
\mathbb{R}_{A}\left(x_{2 i+1}\right)-\mathbb{R}_{A}\left(x_{2 i}\right)=2^{-\mathbb{R}_{A}(a)}+2^{-\mathbb{R}_{A}\left(x_{2 i}\right)}-2^{-\mathbb{R}_{A}(a)}-2^{-\mathbb{R}_{A}\left(x_{2 i-1}\right)},
$$

and since, by the above result, $2^{-\mathbb{R}_{A}\left(x_{2 i}\right)}-2^{-\mathbb{R}_{A}\left(x_{2 i-1}\right)}<\left(\mathbb{R}_{A}\left(x_{2 i-1}\right)-\mathbb{R}_{A}\left(x_{2 i}\right)\right) / 2$, it follows that

$$
\lim _{i \rightarrow \infty}\left(\mathbb{R}_{A}\left(x_{2 i+1}\right)-\mathbb{R}_{A}\left(x_{2 i}\right)\right)=0 .
$$

At this point, on the one hand we can conclude that there exists a unique real $r$ such that

$$
\lim _{i \rightarrow \infty} \mathbb{R}_{A}\left(x_{2 i+1}\right)=r=\lim _{i \rightarrow \infty} \mathbb{R}_{A}\left(x_{2 i}\right),
$$

and, on the other hand, we can say that if, for example, $s \in\left(\mathbb{R}_{A}\left(x_{2 i}\right), \mathbb{R}_{A}\left(x_{2 i+2}\right)\right)$, then $2^{-\mathbb{R}_{A}(a)}+2^{-s} \in\left(\mathbb{R}_{A}\left(x_{2 i+3}\right), \mathbb{R}_{A}\left(x_{2 i+1}\right)\right)$. More precisely,

(1) if $s \leqslant r$ then $2^{-\mathbb{R}_{A}(a)}+2^{-s} \geqslant r$, and

(2) if $s \geqslant r$ then $2^{-\mathbb{R}_{A}(a)}+2^{-s} \leqslant r$.

From the above we have that $r \leqslant 2^{-\mathbb{R}_{A}(a)}+2^{-r} \leqslant r$ and hence $r=2^{-\mathbb{R}_{A}(a)}+2^{-r}$. That is $r$ is the unique solution of 14 .

The reader can check that the above argument easily generalises to the case in which a chain of set-theoretic equation defining a tuple of hypersets is taken into account. That is, if we consider $n$ pairwise distinct hypersets $a_{1}, \ldots, a_{n} \in \mathrm{HF}^{1 / 2}$, the $n$ (again, pairwise distinct) hypersets satisfying

$$
\left\{\begin{array}{ccc}
x^{1} & = & \left\{a_{1}, x^{2}\right\} \\
x^{2} & = & \left\{a_{2}, x^{3}\right\} \\
\vdots & & \vdots \\
x^{n} & = & \left\{a_{n}, x^{1}\right\}
\end{array}\right.
$$

admit codes $\mathbb{R}_{A}\left(x^{1}\right), \ldots, \mathbb{R}_{A}\left(x^{n}\right)$, that are pairwise distinct as well.

Further partial results on our conjectures are mostly related with a study of the codes of the elements of the following sub-family of HF:

Definition 7. The elements of the family $\mathcal{S}$ of super-singletons

$$
\mathcal{S}=\left\{\{\emptyset\}^{i} \mid i \in \mathbb{N}\right\},
$$

are defined recursively as follows: $\{\emptyset\}^{0}=\emptyset$ and $\{\emptyset\}^{n+1}=\left\{\{\emptyset\}^{n}\right\}$. 
Super-singletons were first introduced by Zermelo in [Zer08] as a set-theoretic representation for natural numbers and they have been recently discussed by Kirby in $[\operatorname{Kir} 13]$. The $\mathbb{R}_{A}$-code of super-singletons is easily determined and seen to converge to the above defined value $\boldsymbol{\Omega}$ (see the next proposition). The following figure shows the disposition of the first few code values of super-sigletons (let $s_{i}$ denote the code of the $i$-th super-singleton).

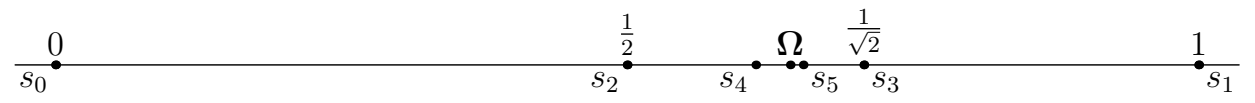

FiguRE 4 . The $\mathbb{R}_{A^{-}}$code of the first 5 super-singletons

As a particular case of the above argument we have that the following holds.

\section{Proposition 5.4.}

$$
0=s_{0}<s_{2}<\cdots s_{2 i}<s_{2 i+2} \cdots<\boldsymbol{\Omega}<\cdots s_{2 i+3}<s_{2 i+1} \cdots<s_{3}<s_{1}=1,
$$

and

$$
\lim _{i \rightarrow \infty} s_{2 i}=\lim _{i \rightarrow \infty} s_{2 i+1}=\mathbf{\Omega} .
$$

With some extra observations and using the above result we can prove the injectivity of $\mathbb{R}_{A}$ on the codes of arbitrary unions of super-singletons.

Definition 8. Given $j$ pairwise distinct indexes $i_{1}, \ldots, i_{j}$, let $s_{i_{1}, \ldots, i_{j}}$ be the code of $\{\emptyset\}^{i_{1}} \cup \cdots \cup\{\emptyset\}^{i_{j}}$, that is $s_{i_{1}}+\cdots+s_{i_{j}}$.

Moreover, let

$$
\mathcal{S}_{i_{1}, \ldots, \overline{i_{j}}}=\left\{s_{i_{1}, \ldots, i_{j}, k} \mid k>i_{j}\right\} .
$$

On the grounds of the above definition, we have that the codes of non-null supersingletons in $\mathcal{S}$ are in $\mathcal{S}_{\overline{0}}$. If we imagine (codes of) super-singletons in $\mathcal{S}_{\overline{0}}$ as resulting from the intersection of a spiral with the $x$-axis, as shown in Figure 5 , then the

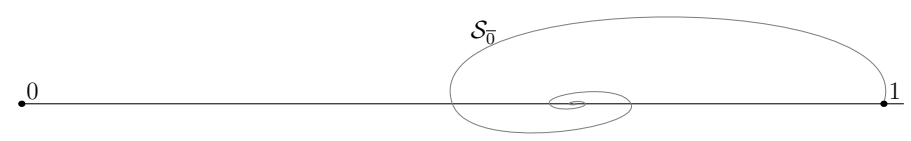

Figure 5. The spiral of $\mathcal{S}_{\overline{0}}$

arrangement of the subsequent spirals $\mathcal{S}_{\bar{i}}$, for $i>0$, can be seen to be a spiral of smaller and smaller spirals, as shown in Figure 6.

Notice that the points of convergence of all the $\mathcal{S}_{\bar{i}}$ 's - that is $\Omega+s_{i}=\mathbb{R}_{A}(\Omega \cup$ $\{\emptyset\}^{i}$ ), for $i \geqslant 0$-are, in fact, codes of hypersets. This is not the case for the point of convergence of all the $\boldsymbol{\Omega}+s_{i}$ 's, that turns out to be $2 \boldsymbol{\Omega}$. Looking at the point 


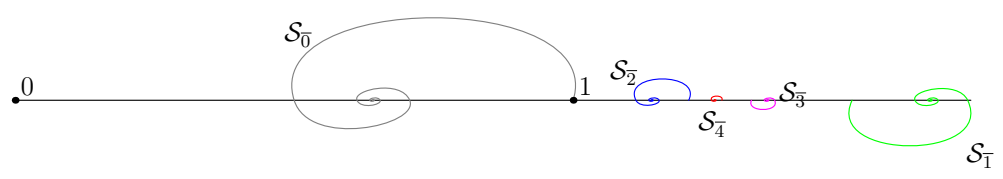

FIGURE 6 . The spirals of $\mathcal{S}_{\overline{0}}, \mathcal{S}_{\overline{1}}, \mathcal{S}_{\overline{2}}, \mathcal{S}_{\overline{3}}, \mathcal{S}_{\overline{4}}$

of convergence of $2^{-\left(\boldsymbol{\Omega}+s_{i}\right)}$ for $i \geqslant 0$, one obtains the sequence of $s_{i+1} \boldsymbol{\Omega}$ that-no wonder-converges to $\Omega^{2}$. Starting from the sequence of spirals $\mathcal{S}_{i, \bar{j}}$, whose points of convergence bring us at $3 \Omega$, by exponentiating we get to $\Omega^{3}$, and so on.

Proposition 5.5. If $\left\{i_{1}, \ldots, i_{j}\right\} \neq\left\{h_{1}, \ldots, h_{k}\right\}$, then $\mathcal{S}_{i_{1}, \ldots, \overline{i_{j}}} \cap \mathcal{S}_{h_{1}, \ldots, \overline{h_{k}}}=\emptyset$.

By letting $h_{i}$ be the set belonging to HF whose Ackermann code is $i$ - that is such that $\mathbb{N}_{A}\left(h_{i}\right)=i$-and looking at indexes that do not necessarily belong to the collection of super-singletons (or to sums of such sets), the following result holds.

Proposition 5.6. For all $i \in \mathbb{N}$ :

(1) $\mathbb{R}_{A}\left(h_{i}\right) \neq \mathbb{R}_{A}\left(h_{i+1}\right)$;

(2) $\mathbb{R}_{A}\left(h_{i}\right) \neq \mathbb{R}_{A}\left(h_{i+2}\right)$.

We can prove the above proposition by rather ad-hoc arguments based on the specific value that a difference between two subsequent codes can assume.

\section{Conclusions and Future Work}

A key point in the construction of our bijection has been the notion of rank, but other rank notions might lead to encodings more satisfactory from a logicomathematical perspective, and along the same lines.

Among the multiple uses of Ackermann's encoding, there is, not surprisingly, combinatorial enumeration. Peddicord [Ped62] obtained a recurrence relation for the number of transitive well-founded sets with $n$ elements via an Ackermannlike bijection between these sets and integer-component vectors $\left(x_{0}, x_{1}, \ldots, x_{n-1}\right)$, where $x_{0}=0$ and $x_{i-1}<x_{i}<2^{i}$, for $1 \leqslant i<n$. Our work can be regarded as a step towards finding the number of graphs on $n$ nodes whose only bisimulation is identity, a problem which Peddicord solved only for acyclic instances.

Another application of Ackermann's bijection is in algorithmics. The best known bound on the time complexity of computing bisimilarity on a graph $G=(V, E)$ is $O(|E| \log |V|)$, cf. [PT87]. However, when the input graph is acyclic, the problem can be solved by an $O(|E|)$-algorithm [DPP04], deep-rooted in Ackermann's order of well-founded sets. In the case of hypersets, we have done the opposite: we employed an algorithmic concept to define an order. It is of interest whether new insight on the said complexity issue can be gained thereby. A real challenge, we believe, lies in the design of 'light' encoding techniques that enable one to shift algorithms from one realm (e.g. integers) to another (e.g., special classes of graphs).

Descriptive complexity issues, a main focus in the study of linear orderings of the universe of hypersets carried out in [LS99], have not been taken into account in this paper; they will be the subject of future work. 
Acknowledgments. The authors wish to thank D. Cantone for useful discussions, suggestions, and contributions on the material in Sec. 5. A.T. acknowledges support from the Academy of Finland under grant 274977.

\section{REFERENCES}

[Ack37] W. Ackermann, Die Widerspruchfreiheit der allgemeinen Mengenlehre, Mathematische Annalen 114 (1937), 305-315.

[Acz88] P. Aczel, Non-well-founded sets, vol. 14 of CSLI Lecture Notes, Stanford, CA, 1988.

[ADR99] D. Aliffi, A. Dovier, and G.-F. Rossi, From set to hyperset unification, The Journal of Functional and Logic Programming (1999), no. 10, 1-48.

[BM91] J. Barwise and L. Moss, Hypersets, The Mathematical Intelligencer 13 (1991), no. 4, 31-41.

[BM96] J. Barwise and L. S. Moss, Vicious circles, CSLI Lecture Notes, Stanford, CA, 1996.

[CNACO08] D. Cantone, M. Nicolosi Asmundo, C. Chiaruttini, and E. G. Omodeo, Cumulative hierarchies and computability over universes of sets, Le Matematiche LXIII, Fasc. I (2008), 31-84.

[DOP99] A. Dovier, E. G. Omodeo, and A. Policriti, Solvable set/hyperset contexts: II. A goaldriven unification algorithm for the blended case, Applicable Algebra in Engineering, Communication and Computing 9 (1999), no. 4, 293-332.

[Dov14] A. Dovier, Set Graphs VI: Logic Programming and bisimulation, CILC 2014 Italian Conference on Computational Logic (Torino, June 16-18, 2014), CEUR Workshop Proc., vol. 1195, L. Giordano, V. Gliozzi, and G.-L. Pozzato, eds., 2014, pp. 14-29.

[DPP04] A. Dovier, C. Piazza, and A. Policriti, An efficient algorithm for computing bisimulation equivalence, Theor. Comput. Sci. 311 (2004), no. 1-3, 221-256.

[DPR06] A. Dovier, E. Pontelli, and G.-F. Rossi, Set unification, Theor. Pract. Log. Prog. 6 (2006), no. 6, 645-701.

[DPR08] Agostino Dovier, Carla Piazza, and Gianfranco Rossi, A uniform approach to constraint-solving for lists, multisets, compact lists, and sets, ACM Trans. Comput. Log. 9 (2008), no. 3.

[FH83] M. Forti and F. Honsell, Set theory with free construction principles, Annali Scuola Normale Superiore di Pisa, Classe di Scienze IV (1983), no. 10, 493-522.

[GPP03] R. Gentilini, C. Piazza, and A. Policriti, From bisimulation to simulation: Coarsest partition problems, J. Autom. Reasoning 31 (2003), no. 1, 73-103.

[Kir13] L. Kirby, Ordinal operations on graph representations of sets, Math. Log. Q. 59 (2013), no. 1-2, 19-26.

[Lev79] A. Levy, Basic Set Theory, Perspectives in Mathematical Logic, Springer-Verlag, 1979.

[LS99] A. Lisitsa and V. Sazonov, Linear ordering on graphs, anti-founded sets and polynomial time computability, Theoretical Computer Science 224 (1999), 173-213.

[OP95] E. G. Omodeo and A. Policriti, Solvable set/hyperset contexts: I. Some decision procedures for the pure, finite case, Comm. Pure Appl. Math. 48 (1995), no. 9-10, 1123-1155, Special issue in honor of J.T. Schwartz.

[Ped62] R. Peddicord, The number of full sets with $n$ elements, Proc. Amer. Math. Soc 13 (1962), 825-828.

[PP04] C. Piazza and A. Policriti, Ackermann Encoding, Bisimulations, and OBDDs, Theory and Practice of Logic Programming 4 (2004), no. 5-6, 695-718.

[PT87] R. Paige and R. E. Tarjan, Three partition refinement algorithms, SIAM J. Comput. 16 (1987), no. 6, 973-989.

[Tar24] A. Tarski, Sur les ensembles fini, Fundamenta Mathematicae VI (1924), 45-95.

[TG87] A. Tarski and S. Givant, A formalization of Set Theory without variables, Colloquium Publications, vol. 41, American Mathematical Society, 1987.

[vN25] J. von Neumann, Eine Axiomatiserung der Mengenlehre, J. für die reine und angewandte Mathematik (1925), no. 154, 219-240, reprinted in [?, pp. 219-240].

[vN61] Collected works vol. I: Logic, Theory of Sets and Quantum Mechanics, Pergamon Press, New York, 1961.

[Zer08] E. Zermelo, Untersuchungen über die Grundlagen der Mengenlehre. I, Mathematische Annalen 65 (1908), no. 2, 261-281 (German). 
Dipartimento di Matematica e Informatica Università di Udine, Via delle Scienze, 206, 33100 Udine, ITALY

E-mail address: giovanna.dagostino@uniud.it

Dipartimento di Matematica e Geoscienze, Università di Trieste, Via Valerio, 12/1, 34127 TRIESTE, ItALy

E-mail address: eomodeo@units.it

Dipartimento di Matematica e Informatica Università di Udine, Via delle Scienze, 206, 33100 Udine, ItALY

E-mail address: alberto.policriti@uniud.it

Helsinki Institute for Information Technology Hitt, Department of Computer Science, University of Helsinki, P.O. 68 (Gustaf Hällströmin katu 2B), FI-00014 - Helsinki, FinLAND

E-mail address: tomescu@cs.helsinki.fi 